\title{
Introduction to Customer Analytics and Data-Led Omnichannel Commerce Minitrack
}

\author{
Petri Parvinen (University of Helsinki, Finland) \\ Maurits Kaptein (University of Tilburg, the Netherlands) \\ Juho Hamari (University of Tampere, Finland) \\ Essi Pöyry (Aalto University, Finland)
}

The continued development of e-commerce, social media, online information search and business intelligence have fundamentally changed the conducting of business in all fields and industries. Virtually all businesses are now facing an omnichannel world, in which presence and activity in a number of different channels determines business success. The rise of digital communications and data has changed many things in traditional industries as more and more industries are able to understand, track, serve and sell their offerings digitally. Passing customers smoothly from one channel to another is increasingly important, and many businesses that have excelled in the digital space are turning to more traditional channels to embrace the combination opportunities. Simultaneously new media and applications, e.g. cutting edge moving image social media applications, commoditized high-definition virtual reality and algorithm-based automated procurement systems, keep the scene lively. Combining the old and the new has become a competence and a sustainable source of competitive advantage. One of the primary sources of this competitive advantage is the ability to extract timely and relevant customer data for serving customers better. Customer analytics comes in various forms, but the cutting edge of such analytics is one that starts from practical and acute needs of the customer. Such customer analytics is not legacy database-led, but deals with customer interaction, engagement and service design, and is linked to protocols of how to react to, target, automate and optimize. A minitrack concentrating on these core developments of ecommerce and e-business is proposed for HICSS- 50 .

Research on cutting edge customer analytics and omnichannel marketing requires awareness of the realities and possibilities of information systems management. At marketing-centric venues such as pure marketing and even e-commerce conferences, the starting point is often too much on the would-benice-to-have or who-uses-it-the-most type of dialogues. Marketing research without information systems understanding also ignores the fact that many of the developments are and will continue to be technology-pushed, and that many of these technologies emerge from IS, computer science and human-computer interaction disciplines. Implementation and the management of the systems and infrastructure can also be mistakenly taken as granted. HICSS represents the solution to all of these issues. More importantly, the proposed minitrack can be of interest to many HICSS attendees due to its combination of economic relevance, familiar business contexts and cutting edge technology.

The minitrack encouraged submissions from both cutting edge technology and business application. Fields of research that have been used to contribute to the minitrack included e.g. e-commerce, social commerce, online persuasion, behavior change, selling through social media, electronic word-ofmouth, automated customer response systems, collaborative technology, online customer service, salesforce and sales support digitalization, recommender applications and interactive persuasive technologies This year the minitrack at HICSS attracted a good number of papers and the ones chosen for the conference included relevant topics like:

- The adoption of mobile (vs. PC) e-commerce in different purchase categories

- Utilitarian vs. hedonic motivations for technology usage

- Personalization and intrusiveness of product recommendations 